\title{
The coexistence of odd and even parity magnetic fields in disc galaxies
}

\author{
D. Moss ${ }^{1}$ and D. Sokoloff ${ }^{2}$ \\ 1 School of Mathematics, University of Manchester, Oxford Road, Manchester, M13 9PL, UK \\ e-mail: moss@ma.man.ac.uk \\ 2 Department of Physics, Moscow University, 119992 Moscow, Russia
}

Received 19 March 2008 / Accepted 30 May 2008

\section{ABSTRACT}

\begin{abstract}
Aims. Naive dynamo models predict that large-scale magnetic fields generated in flattened disc-like structures will be steady and symmetric with respect to the equatorial plane, whereas fields generated in quasi-spherical volumes will be oscillatory and anti-symmetric. Spiral galaxies consist of a flattened disc and a quasi-spherical halo. We thus investigate to what extent this naive understanding of symmetry properties is realised in composite disc/halo models for galactic magnetic fields.

Methods. We consider generation of galactic magnetic fields in the framework of galactic mean field dynamo theory, based on the effects of differential rotation and helical turbulent motions (the " $\alpha$-effect"), using conventional profiles for both generators of magnetic field in the disc and halo. The halo and disc regions are mostly separated by a substantial contrast between their turbulent diffusivities, respectively $\eta_{\mathrm{d}}$ and halo $\eta_{\mathrm{h}}$. We solve the corresponding equations of mean field electrodynamics numerically, using contrasts up to $\eta_{\mathrm{h}} / \eta_{\mathrm{d}}=5$, while realizing that it might be realistic to consider significantly larger values.

Results. In contrast to our naive expectations coexisting steady symmetric (quadrupole-like) magnetic structures in the disc and oscillating antisymmetric (dipole-like) structures in the halo were not found. Usually one component of the dynamo system enslaves the other: a more dynamo-active disc creates a symmetric field in the halo as well as in the disc or, conversely, a more dynamo-active halo generates antisymmetric magnetic fields that pervade both halo and disc. Our most interesting models are mixed parity solutions at the transition between the two regimes.

Conclusions. We consider the results obtained as presenting a challenge for the contemporary theory of galactic magnetic fields. We note that there is some recent observational evidence for a difference in symmetry properties between disc and halo. We see three possible resolutions of the problem. The contrast $\eta_{\mathrm{h}} / \eta_{\mathrm{d}}$ used may have been too low (values up to about 50 can be argued for but implementation of such values is rather demanding numerically). Another option is that galactic magnetic structures may be separated in two distinct classes, namely objects with disc-like and halo-like magnetic field structures. To settle this issue observationally seems to require much higher resolution than is possible with telescopes now available. Finally, of course, can not be excluded that something fundamental is lacking from our understanding of the origin of galactic magnetic fields.
\end{abstract}

Key words. galaxies: magnetic fields - Galaxy: disk - ISM: magnetic fields

\section{Introduction}

There is a near consensus among dynamo theorists that the largescale magnetic field in disc galaxies should be of even parity with respect to the disc midplane. This is based on the finding that the most readily excited dynamo mode in thin disc geometry is of this type (i.e. "quadrupole-like"), see e.g. Ruzmaikin et al. (1988). Although established originally for strictly thin disc models (with boundary conditions applied at $z= \pm h, h / R \ll 1$, where $z$ is height above disc, and $h$ and $R$ are disc semi-thickness and radius respectively), the result also appears to apply to the simplest "embedded disc" models (e.g. Moss \& Brandenburg 1992), in which the dynamo-active disc is surrounded by a quasispherical halo of higher magnetic diffusivity.

Even so, there have been challenges to this consensus. Sokoloff \& Shukurov (1990) observed that whereas the galactic disc would be expected to support a quadrupole-like magnetic field, a dynamo operating in the quasi-spherical halo should generate a field of odd ("dipole-like") symmetry, and that the interaction between these two components was potentially of interest. These ideas were explored numerically to some extent by Brandenburg et al. (1992), but they only found quadrupolelike stable fields, which permeated both disc and halo, i.e. the disc dynamo was dominant. Earlier, Moss \& Tuominen (1990), whilst not disputing that the ultimate state of a galactic dynamo would have even parity, showed that transients with intermediated ("mixed") parity might be sufficiently long-lived to be of interest.

Observations of external galaxies at best give hints as to the symmetry properties with respect to the midplane of their fields. The small number of well-observed systems that are viewed edge-on do seem to host quadrupole-like fields, but the evidence is not completely conclusive (see, e.g., Beck et al. 1996).

At the same time, attempts have been made to determine the large-scale structure of the Milky Way field. This is in some ways more difficult, as local small-scale features can confuse interpretation of observations (there is, for example, still dispute about the number of reversals of the disc field with radius). The most useful measurements are those of rotation measures (RMs) of pulsars (preferentially distributed at low galactic latitudes) and extra-galactic sources (more uniformly distributed in latitude). Pulsar studies seem to indicate a quadrupolar symmetry near the plane (e.g. Frick et al. 2001), but other work (e.g. Han et al. 1997; Han 2006; and Han, pers. comm. 2007) based on RMs of extra-galactic sources suggests that the field at high 
galactic latitudes might be of odd parity. Recently Sun et al. (2008) gave a convincing analysis of polarization and rotation measure data, and came to the conclusion that a large-scale magnetic field reversal is needed between the halo field above and below the galactic equator whereas the disc field has the same direction above and below the galactic equator. This appears to be consistent with the suggestion of Sokoloff \& Shukurov (1990).

This is an intriguing prospect, and provides challenges for the dynamo origin of galactic magnetic fields. Moss \& Shukurov (2001) studied the interaction between an accreted field of scale significantly larger than that of the galaxy and a conventional galactic dynamo. They found fields of intermediate parity for quite a wide range of parameters. Whether or not such fields are consistent with the above results has not been established.

Given all these uncertainties and possibilities, we return here to the study of the interaction between disc and halo dynamos, inter alia in an attempt to clarify whether solutions that are quadrupole-like near the disc plane and dipole-like in the halo can be found.

Before beginning our analysis we note that our intention is not to give any realistic model of magnetic field generation in a particular (or even a generic) galaxy, but rather to illuminate some unanswered questions and obscure problems in conventional dynamo theory as applied to disc galaxies.

\section{The model}

We solve the standard mean field dynamo equation

$$
\frac{\partial \boldsymbol{B}}{\partial t}=\nabla \times(\boldsymbol{u} \times \boldsymbol{B}+\alpha \boldsymbol{B}-\eta \nabla \times \boldsymbol{B}),
$$

where $\eta$ is the turbulent diffusivity and $\alpha$ represents the usual isotropic alpha-effect. We restrict our studies to axisymmetric solutions. $\boldsymbol{u}=\Omega \varpi \hat{\phi}$, where $\hat{\phi}$ is the unit azimuthal vector and $\Omega$ is the angular velocity.

First of all, note that a simultaneous solution of the dynamo equations in the disc and halo regions is a non-trivial task computationally. In practice we are unable to apply the numerical scheme to what we feel to be a completely realistic choice of the parameters governing dynamo action in the disc and halo; in particular we take a somewhat thicker disc, and a smaller contrast in diffusivity between disc and halo, than might be felt to be ideal, based on observational estimates of length and velocity scales. We do our best to satisfy as far as possible the different requirements needed to describe dynamo action in the disc and quasi-spherical halo, at least with the computational resources available for this exploratory investigation.

We define spherical polar coordinates $(r, \theta, \phi)$, and also cylindrical polar coordinates $(\varpi, \phi, z)$. Our dynamo domain is a sphere radius $R$, with an embedded disc region $|z| \leq d, \varpi \leq \varpi_{\mathrm{d}}$. In the halo at large values of $r, \eta \rightarrow \eta_{\mathrm{h}}$, whereas in the disc domain $\eta=\eta_{\mathrm{d}}$, and there is a transition region of thickness $\delta R$. Similarly, in the disc region $\alpha=\alpha_{0} \sin (\pi z / d)$, and in the halo at large $r, \alpha \rightarrow \alpha_{0} \cos \Theta$, where $\Theta=\cos ^{-1}\left(z-z_{\mathrm{d}}\right) / r_{\mathrm{f}}$, where $r_{\mathrm{f}}^{2}=\left(z-z_{\mathrm{d}}\right)^{2}+\varpi^{2}$, ensuring a smooth transition between disc and halo regions. Latitudinal profiles of $\alpha$ and $\eta$ are shown in Fig. 1.

In the disc region, the angular velocity is given by

$\Omega=\frac{\Omega_{0}}{\left(1+\left(\frac{\varpi}{\varpi_{B}}\right)^{2}\right)^{1 / 2}}$,

with $\varpi_{B}=0.1 R$. In the halo, $\Omega \rightarrow \Omega_{1} / r$ at large $r$, with $\Omega_{1}$ chosen to give continuity of $\Omega$ at $r=r_{\mathrm{d}}, z=z_{\mathrm{d}}$, again with


Fig. 1. Profiles of $\alpha$ (lower) and $\eta$ (upper) at radii $R / 3$ (solid) and $2 R / 3$ (broken) for $R_{\alpha}^{\mathrm{h}}=5, R_{\alpha}^{\mathrm{d}}=40$ and $\eta_{\mathrm{h}} / \eta_{\mathrm{d}}=5 . \theta$ is galactic latitude.



Fig. 2. Contours of angular velocity $\Omega$ in a meridian plane. The small dashed arc represents the inner boundary of the computational domain, and the disc domain is also outlined.

a smooth transition elsewhere between disc and halo. Contours of $\Omega$ are shown in Fig. 2.

Thus in the disc region we have a disc dynamo of the type widely used to model galactic dynamos, whereas the halo region is more akin to a convention spherical dynamo. Nondimensionalization in terms of $\eta_{\mathrm{d}}$ and $R$ yields standard dynamo parameters $R_{\alpha}^{\mathrm{d}} d=\alpha_{\mathrm{d}} R / \eta_{\mathrm{d}}, R_{\alpha}^{\mathrm{h}}=\alpha_{\mathrm{h}} R / \eta_{\mathrm{d}}, R_{\omega d}=\Omega_{0} R^{2} / \eta_{\mathrm{d}}$. We take a diffusivity contrast $\eta_{\mathrm{h}} / \eta_{\mathrm{d}}=5$; this value is as large as we can use reliably with the model without requiring excessive computational resources. (We base the definitions of $R_{\alpha}$ and $R_{\omega}$ 
Table 1. Summary of results. In all cases $\eta_{\mathrm{h}} / \eta_{\mathrm{d}}=5, R_{\omega}=10^{4}, h / R=0.2$. SP, DP denote respectively singly periodic and doubly periodic solutions. $\mathrm{M}(\mathrm{eq})$ means migration in the equatorward direction. $P$ is global parity, so $P=1$ denotes a quadrupole-like solution, $P=-1$ a dipole-like solution. $E$ is the global magnetic energy.

\begin{tabular}{cccl}
\hline \hline Model & $R_{\alpha}^{\mathrm{d}}$ & $R_{\alpha}^{\mathrm{h}}$ & Solution \\
\hline 101 & 5 & 0 & $P \rightarrow 1$, steady, disc dominated \\
102 & 0 & 150 & osc, M(eq) outside of disc \\
104 & 3 & 2 & steady decay \\
$105 \mathrm{~b}$ & 3 & 10 & steady, $P=+1$ \\
$106 \mathrm{~b}$ & 3 & 20 & steady, $P=+1$ \\
$153 \mathrm{~b}$ & 3 & 25 & steady, $P=+1$ \\
$154 \mathrm{~b}$ & 3 & 30 & decay \\
$155 \mathrm{~b}$ & 3 & 35 & slow approach to steady $P=+1$ through oscillatory transients. Disc dominated \\
$107 \mathrm{~b}$ & 3 & 40 & SP in E, DP in $P, P \rightarrow-1$ slowly, halo dominated, M(eq) at large radii, little migr. at small radii \\
108 & 3 & 60 & oscillatory in $P$ and $E$ \\
109 & 3 & 200 & oscillatory in $P$ and $E$ \\
$131 \mathrm{~b}$ & 5 & 2 & $P=+1$, steady, disc dominated \\
$132 \mathrm{~b}$ & 5 & 10 & $P=+1$, steady, disc dominated \\
$133 \mathrm{~b}$ & 5 & 20 & $P=+1$, steady,mainly disc dominated, some significant $B_{\text {tor }}$ in halo \\
$138 \mathrm{~b}$ & 5 & 35 & slow approach to steady $P=+1$ through oscillatory transients. \\
$139 \mathrm{~b}$ & 5 & 37 & DP in $P, 0.993 \leq P \leq 0.995$, SP in $E$ \\
$134 \mathrm{~b}$ & 5 & 40 & SP, $0.95 \leq P \leq 0.97$, disc dominated, some field in halo \\
$135 \mathrm{~b}$ & 5 & 60 & DP in $\mathrm{P}, 0.1 \leq P \leq 0.6 \mathrm{SP}$ in $\mathrm{E}$, field mainly in halo, M(eq) in halo \\
$136 \mathrm{~b}$ & 5 & 200 & SP in $\mathrm{P}, 0.55 \leq P \leq 0.80, B_{\text {pol }}$ in halo, some $B_{\text {tor }}$ also in disc, M(eq) \\
\hline
\end{tabular}

on the disc radius $R$, which is more convenient for the halo. In principle one could use here the disc half-thickness $h$ for the length scale, and this indeed is the standard choice for discdominated dynamos; one definition can be easy converted to the other - see below.) A representative sample of the models investigated is summarized in Table 1. The parameter range covered by this table covers the estimates for dynamo governing parameter that look relevant for disc and halo and, for the purposes of illustration, extends beyond this realistic range. We need to explore this wide range in parameter space to demonstrate that the negative result reported would not be overturned by a revision in estimates for parameters. Note that if the definition of $R_{\alpha}$ and $R_{\omega}$ were based on the disc half-thickness $h$ rather on the galactic radius $R$, as above, then the values would be reduced by a factor $h / R \approx 1 / 5$, and $(h / R)^{2} \approx 1 / 25$ respectively. Also the effective values of these parameters in the halo are reduced by a factor $\eta_{\mathrm{d}} / \eta_{\mathrm{h}}$. For real galaxies, $h / R$ can be as low as $1 / 50$ (e.g. disc half-thickness $h=0.4 \mathrm{kps}, R=20 \mathrm{kpc}$ ) and $\eta_{\mathrm{d}} / \eta_{\mathrm{h}}$ can be as low as $1 / 50$. Then typical values for $R_{\omega} R_{\alpha}$ become more of order 10 and 1, i.e. nearer to the conventional estimates used in disc dynamo models (e.g. Ruzmaikin et al. 1988).

\section{Results}

The results of our numerical modeling are presented in Table 1 as well as in Figs. 3-11. Note that numerical investigation of the multidimensional parameter space and isolation of the important governing parameters is far from straightforward. It would be impractical to present here all the models explored. Reflecting this, and to avoid possible future confusion, we retain here the enumeration of the models from the contemporary log.

We see from Table 1 that the domain of dynamo activity, i.e. disc or halo, where dynamo action is stronger (determined by the magnitude of the dynamo number $D=R_{\alpha} R_{\omega}$ ) dominates and enslaves the less active volume. (It should be remembered that the dynamo numbers $R_{\alpha}$ and $R_{\omega}$ are defined in terms of the disc diffusivity $\eta_{\mathrm{d}}$, but the halo diffusivity is substantially larger.) We classify the type of configuration based on the symmetry of the magnetic configuration and its time behaviour, i.e. we consider quadrupolar steady solutions as disc-like while dipolar


Fig. 3. Snapshot of poloidal field lines (left hand panel) and toroidal field contours (right hand panel) in a meridian plane for the halo dominated model $135 \mathrm{~b}$ - see Table 1 .

oscillating solutions are considered as halo-like solutions. We consider the disc region to be enslaved by the halo when the field behaviour in the disc is oscillatory, and the halo to be enslaved by the disc when its field is steady. That is, the disc/halo is enslaved by the halo/disc when the behaviour of its magnetic field is not typical of a disc/halo dynamo, but resembles that of a dynamo in the complementary region.

We present results for model $135 \mathrm{~b}$ as an example of halo-like structures (Figs. 3 and 5) and those for model 133b (Fig. 6) as an example of the disc-like structures. We only show the time behaviour (Fig. 5) of a halo-like structure because the disc-like structures are steady. In Fig. 7 we show the parities and energies of the fields in the disc and halo regimes separately. Both parities oscillate, with mean significantly smaller than +1 , demonstrating the dominance of the halo field as $R_{\alpha}^{\mathrm{h}}$ becomes large. The upper 
(a)

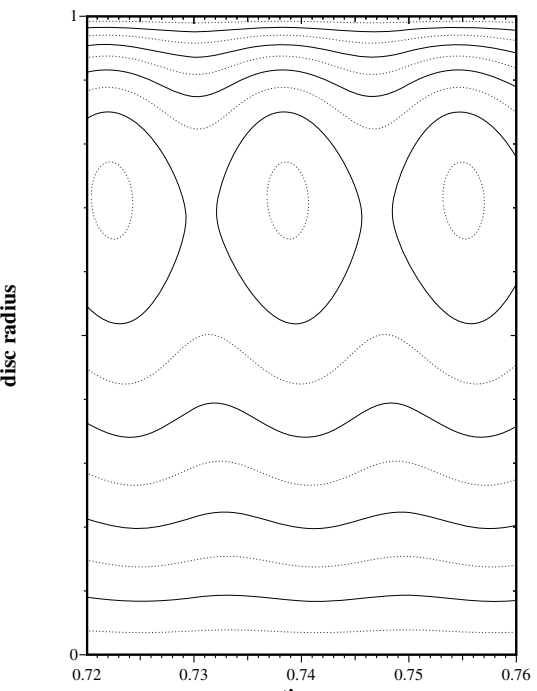

(b)


(c)

Fig. 4. Model 134b: space-time ("butterfly") diagrams for a) the azimuthal field in the equatorial plane - the vertical axis is distance from the rotation axis; b) the azimuthal field in the halo at distance $0.98 R$ from the origin; c) the azimuthal field at radius $0.15 R$. In cases $\mathbf{b}$ ) and c) the vertical axis is the angle $\theta$.
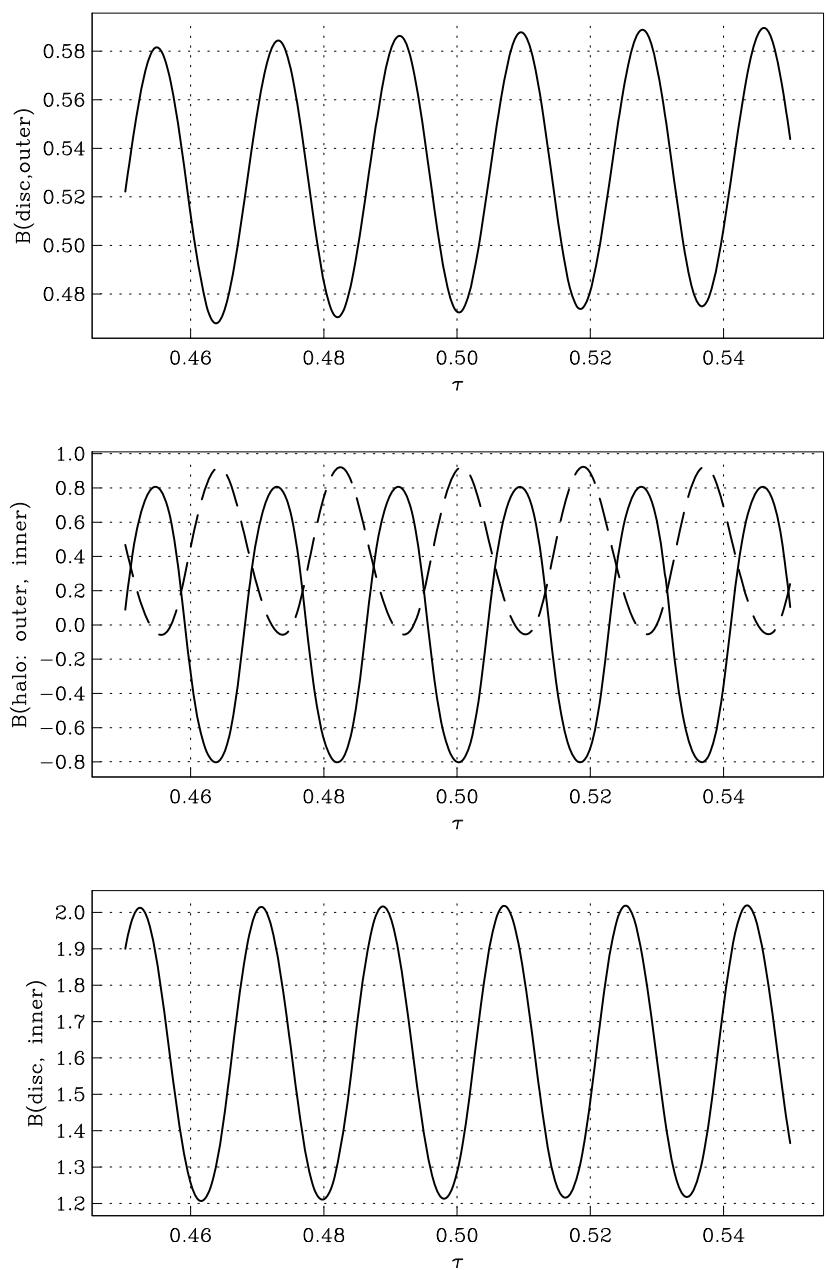

Fig. 5. Model 135b: time dependence of $B_{\phi}$ in inner and outer parts of the disc region at $r=0.1 R, \theta=0.495 \pi$ and $r=0.9 R, \theta=0.495 \pi$ (bottom and top panels respectively), and (middle panel) in the inner and outer parts of the halo region at $r=0.1 R, \theta=0.25 \pi$ and $r=$ $0.9, \theta=0.25 \pi$ (broken and continuous curves respectively).


Fig. 6. Snapshot of poloidal field lines (left hand panel) and toroidal field contours (right hand panel) for the disc dominated model 133bsee Table 1 . 



Fig. 7. Model 135b: in the lower panel, the time dependence of the parity of the disc fields (solid) and halo field (broken) curves, in the upper panel the behaviour of the corresponding magnetic energies.

panel shows the corresponding field energies; those of the disc and halo regions are comparable and oscillate in phase.

Various transient states between these two extreme regimes can be found. The results for model $134 \mathrm{~b}$ are presented as an illustrative example of such a state in Figs. 4 (space-time diagram) Fig. 8 (temporal behaviour) and Fig. 9 (field contours). In Fig. 10 we show the behaviour of the parities and energies in the disc and halo regions. Here the disc parity is close to $P=+1$, and is almost constant, whereas the halo parity has a significantly lower value and oscillates. The upper panel shows the disc and halo field energies; the disc energy is here larger than that of the halo. This behaviour can be contrasted with that of model $135 \mathrm{~b}$ described above, for an only slightly larger value of $R_{\alpha}^{\mathrm{h}}$.

We see that we can find quite complicated temporal behaviour and nontrivial spatial structure which is however quite remote from the naive expectation and hardly can be described as a combination of disc- and halo-like structures.

Note that the contrast in the governing parameters for the models $133 \mathrm{~b}$ and $135 \mathrm{~b}$ is quite modest, i.e. $R_{\alpha}$ for the halo differs by factor of about 3 from one model to the other. It would be unrealistic to claim that contemporary astronomy can estimate this quantity to such accuracy. The point is that until now no direct observations of the $\alpha$-effect in galaxies are available. Even calculation of the magnitude of the $\alpha$-effect from detailed numerical simulations of galactic MHD is a highly nontrivial business (Brandenburg \& Sokoloff 2002; Otmianowska-Mazur et al. 2007) because of substantial fluctuations in the $\alpha$-coefficient. Solar observations of tracers for $\alpha$-effect (e.g. Xu et al. 2007) as well as laboratory measurements (Stepanov et al. 2006) whilst being intrinsically important are obviously too preliminary to be
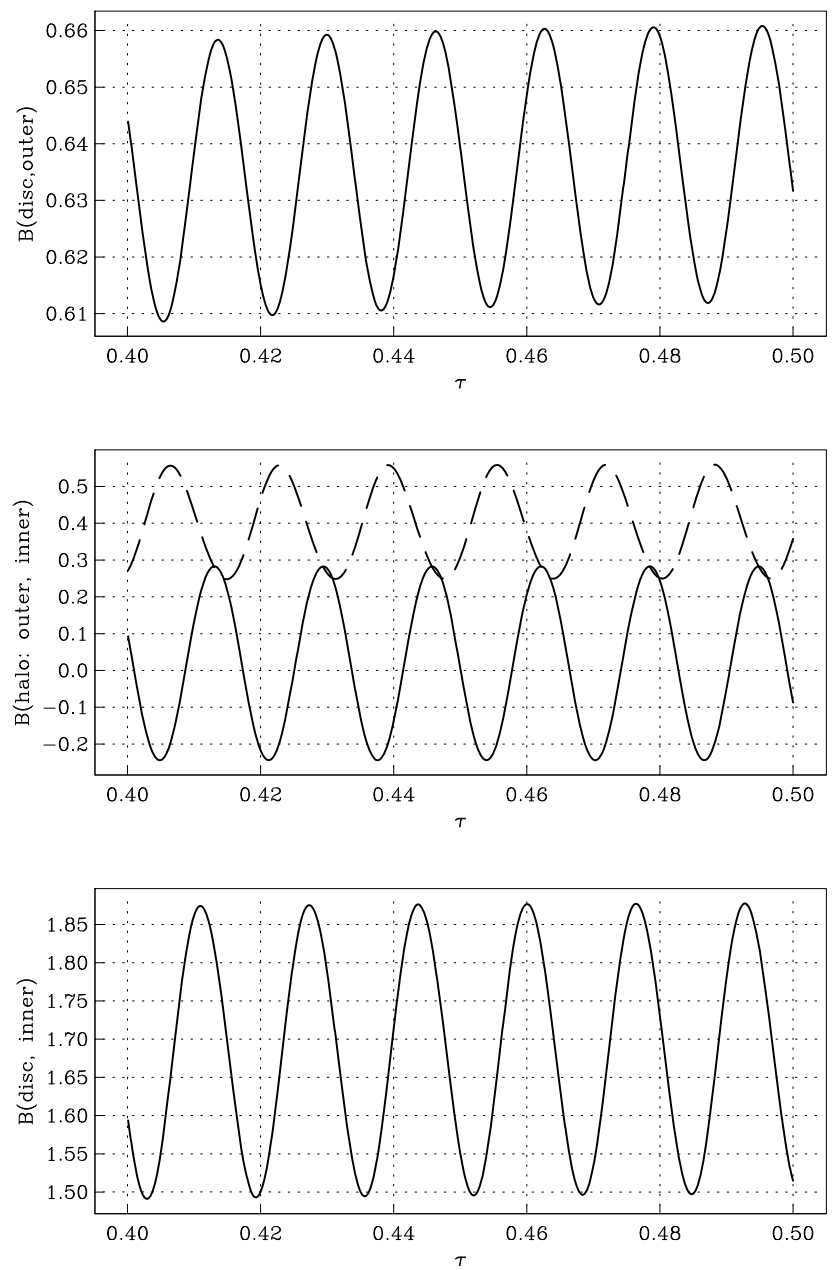

Fig. 8. Model 134b: as Fig. 5, time dependence of $B_{\phi}$ in inner and outer parts of the disc region (bottom and top panels), and (middle panel) in the inner and outer parts of the halo region (broken and continuous curves respectively).

instructive for galactic dynamos. In other words, a moderate tuning of the governing parameters for galactic dynamo can switch the solution from a disc-like structure to a halo-like one. The situation looks in general to be a challenge for observational astronomy.

From Figs. 3 and 6 we can see that if the halo dynamo enslaves the disc region. then the generated magnetic field may penetrate from the halo to the disc effectively. However, it can be seen from Fig. 6 that if the disc enslaves the halo then the penetration of magnetic field from the disc to the halo is rather less effective, It seems that the presence of a substantial regular magnetic field in the halo needs substantial dynamo action there. However when the halo dynamo is very (maybe unrealistically) strong, e.g. model 136b, then the disc field may be very weak compared to that in the halo.

To summarize, it seems hard or impossible to obtain a solution with steady disc and oscillatory halo magnetic fields. We can demonstrate a clear transition from disc-like solutions (steady, e.g. models 133b, 153b) through "mixed" solutions (e.g. model 134b), to halo-like solutions (model 135b, 108). There is the interesting technical point that at the lower disc dynamo number $R_{\alpha}^{\mathrm{d}}=3$, during the transitional interval there are a range of halo dynamo numbers around model 154b where a dynamo is not excited. Such transitions between disc dominated steady configurations and oscillatory halo dominated solutions can be 

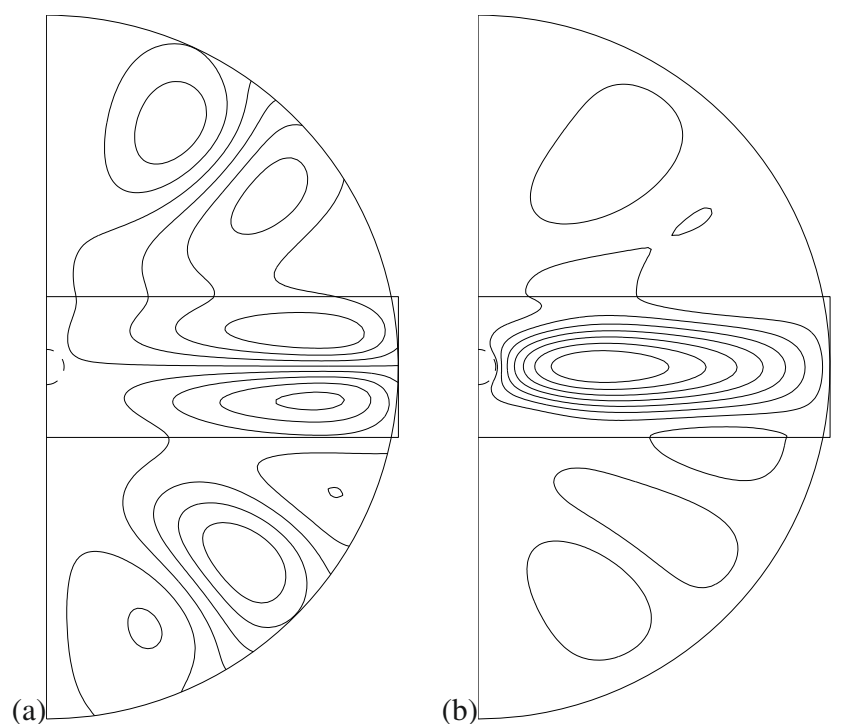

Fig. 9. Snapshot of poloidal field lines (left hand panel) and toroidal field contours (right hand panel) for the intermediate model 134b - see Table 1.
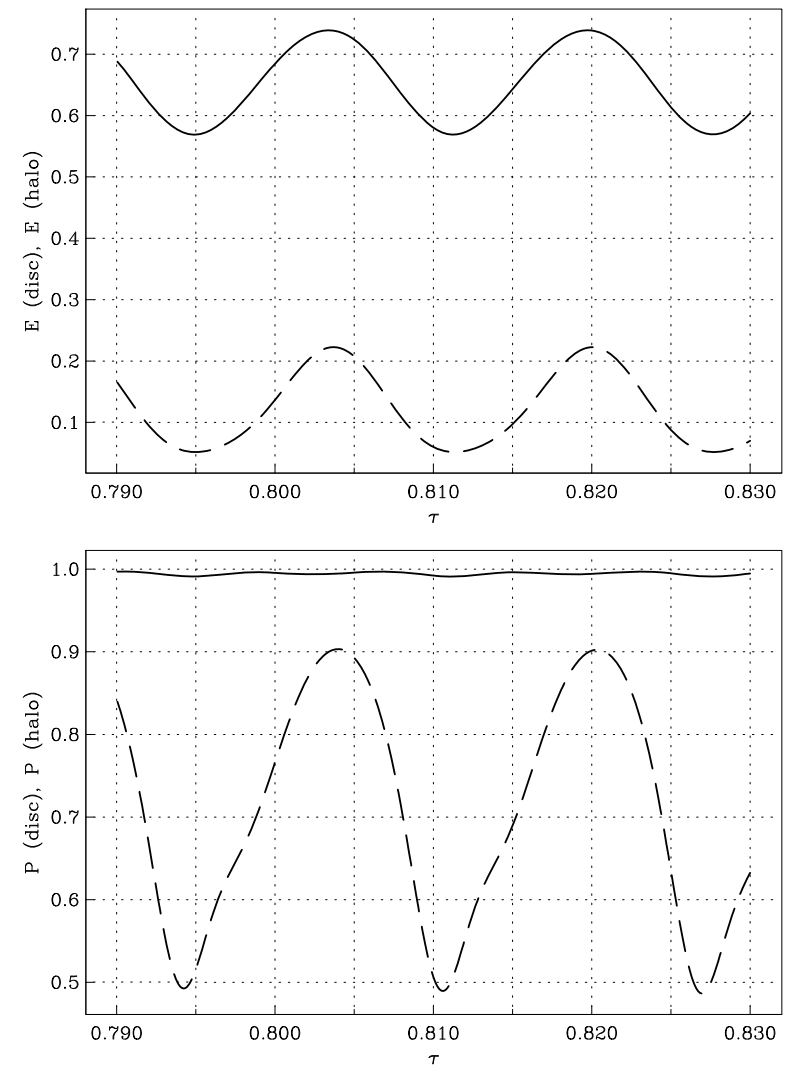

Fig. 10. Model 134b: in the lower panel, the time dependence of the parity of the disc fields (solid) and halo field (broken) curves, in the upper panel the behaviour of the corresponding magnetic energies.

brought about by decreasing $\eta_{\mathrm{h}}$ or equivalently by increasing $\alpha_{\mathrm{h}}$, or by other modifications of the dynamo governing parameters.

We believe that model $134 \mathrm{~b}$ is the closest approach to our speculation of a magnetic field configuration with a "halo-like" solution in the halo and an independent "disc-like" solution in the disc. We thus show additional plots to demonstrate the configuration in more detail (Fig. 11). Here the magnetic configurations in the halo and disc regions do appear to behave quite



(a)

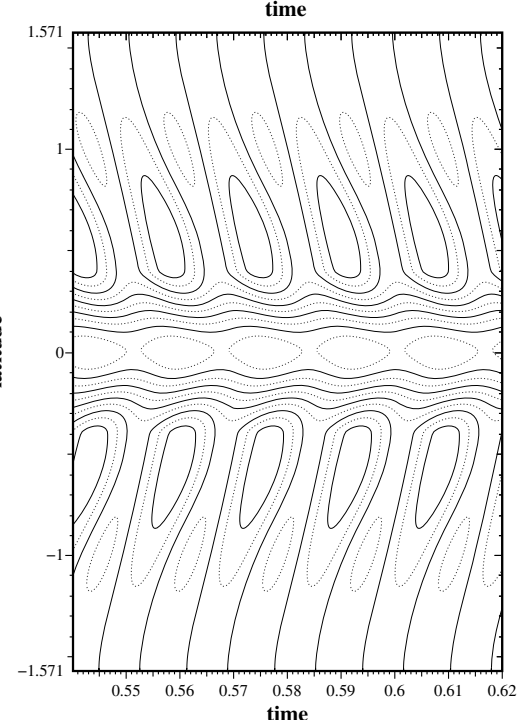

Fig. 11. Magnetic field configuration in the model 134b: a, b - latitudetime diagram for $r=0.75$ and $r=0.5$.

independently. The halo magnetic structure looks like a travelling wave while the disc structure resembles a standing wave or vacillations of magnetic field - but not a stationary solution. Note that even such configurations can only be found for very limited ranges of parameters. Thus our numerical results really do not support our conjectures outlined in Sect. 1. Possibly, we should here remember the wisdom of the Russian philospher Kozma Proutkoff that "Some phenomena remain obscure for us not because of the weakness of our concepts but rather because the phenomena are beyond the scope of the concepts", and so modify our expectations concerning the magnetic configuration in the disc.

\section{Discussion}

The results obtained are in a sense quite discouraging. We were unable to find the solutions of the type anticipated from our naive expectations of galactic magnetic field studies, i.e. dipole-like oscillatory solutions in the halo together with quadrupole-like steady solutions in the disc. In other words, we were unable to develop a dynamo model consistent with the conclusions of Sun et al. (2008). Our most "promising" models, with almost steady disc fields and oscillatory halo fields (e.g. model 134b), can only 
be found for very limited parameter ranges (while the other results presented correspond to "typical" solutions obtained without special adjustment of parameters). Of course, it is difficult to be sure that somewhere in a multi-dimensional parameter space such solutions do not occur. However, given the efforts here and in the other studies cited, this perhaps looks implausible.

We should perhaps comment that the oscillatory nature of solutions could never be directly observable - timescales are typically $O\left(10^{2}\right)$ Myr. Definitive determination of a galactic magnetic field with intermediate parity would be a necessary condition for the existence of such a solution - but not sufficient as steady solutions with intermediate parity are also found. We can envisage two possible resolutions of the problem from the theoretical viewpoint. It may be that we do not understand something fundamental about the galactic dynamo mechanism. On the other hand, we may not have implemented sufficient contrast between the disc and halo - this is a numerical issue. The latter option appears less radical and so more acceptable. In this respect we note that our largest contrast in $\eta_{\mathrm{h}} / \eta_{\mathrm{d}}$ was 5 (this is a result of computational constraints) whilst Sokoloff \& Shukurov (1990) discuss contrasts as large as 50. (Although, as stressed above, estimation of these parameters is very uncertain - significant direct observational input on this issue would be very valuable! The question here is what observational quantities would be useful observational diagnostics for turbulent diffusivity. Naively, estimates of turbulent velocities and length scales would be valuable.) We would need to improve substantially our numerical implementation to achieve such contrasts in $\eta_{\mathrm{h}} / \eta_{\mathrm{d}}$. Concerning the observational aspects of the problem, we feel that the available observations, whilst very interesting and provocative, need substantial development and confirmation before they can confirm or reject quite definitively the existence of distinct magnetic field symmetries in the disc and halo components of galaxies.

One further issue for consideration is that our dynamo model does not include various outflows (e.g. "galactic winds") which would naturally make the magnetic systems in disc and halo more connected with one other (cf. Brandenburg et al. 1993;
Shukurov et al. 2006; Sur et al. 2007). Such an increase in connectivity between disc and halo might be expected to increase the tendency for the enslavement of a less dynamo active component (say, the halo) by the stronger one (the disc say), in opposition to what might be expected from an increase in diffusivity contrast. Thus the failure to produce fields consistent with the Sun et al. (2008) findings would become more marked. It is difficult however to exclude a priori that a particular combination of outflows could not result in a solution of the desired configuration.

Acknowledgements. We are grateful to A. Shukurov for useful discussions, and to the referee, R. Beck, for a number of helpful suggestions. D.S. thanks the Royal Society for financial support of his visit to the UK, and RFBR for financial support under grant 07-02-00127.

\section{References}

Beck, R., Brandenburg, A., Moss, D., Shukurov, A., \& Sokoloff, D. 1996, ARA\&A, 34, 155

Brandenburg, A., \& Sokoloff, D. 2002, Geophys. Astrophys. Fluid, 96, 319 Brandenburg, A., Donner, K. J., Moss, D., et al. 1992, A\&A, 259, 453 Brandenburg, A., Donner, K. J., Moss, D., et al. 1993, A\&A, 271, 36

Frick, P., Stepanov, R., Shukurov, A., \& Sokoloff, D. 2001, MNRAS, 325, 649

Han, J. L. 2006, Chinese J. Astron. Astrophys., Suppl., 6, Issue S2, Proceedings of the 2005 Lake Hanas International Pulsar Symposium, ed. N. Wang, R. N. Manchester, B. J. Rickett, \& A. Esamdin., 211

Han, J. L., Manchester, R. N., Berkhuijsen, E. M., \& Beck, R. 1997, A\&A, 322, 98

Moss, D., \& Brandenburg, A. 1992, A\&A, 256, 371

Moss, D., \& Shukurov, A. 2001, A\&A, 372, 1048

Moss, D., \& Tuominen, I. 1990, Geophys. Astrophys. Fluid Dyn., 50, 113

Otmianowska-Mazur, K., Kowal, G., \& Hanasz, M. 2007, ApJ, 668, 110

Ruzmaikin, A., Shukurov, A., \& Sokoloff, D. 1988, Magnetic Fields of Galaxies (Dordrecht: Kluewer)

Shukurov, A., Sokoloff, D., Subramanian, K., \& Brandenburg, A. 2006, A\&A, 448, L33

Sokoloff, D., \& Shukurov, A. 1990, Nature, 347, 51

Stepanov, R., Volk, R., Denisov, S., et al. 2006, Phys. Rev., E73, 046310

Sun, X. H., Reich, W., Waelkens, A., \& Enßlin, T. A. 2008, A\&A, 477, 573

Sur, S., Shukurov, A., \& Subramanian, K. 2007, MNRAS, 377, 874

Xu, H., Gao, Y., Zhang, H., et al. 2007, Adv. Sp. Res., 39, 1715 